\title{
An Overview of Existing Animal Models of Post- traumatic Stress Disorder Regarding Validity and Ethics
}

\author{
Yijie Lian \\ Steinhardt School of Culture, Education, and Human Development, New York University, New York, NY 10003, \\ America \\ Corresponding author. Email: yl5436@nyu.edu
}

\begin{abstract}
Animal models have historically played a significant role in the overall understanding of the underlying pathology of human diseases, as well as development and validation of novel therapeutic treatments. In the past few decades, they have been heavily used in researches regarding post-traumatic stress disorder (PTSD). While the animal models have remarkably contributed to the field at both genetic and molecular levels, there has been rising conversation questioning the efficacy of commonly used animal models in terms of investigating PTSD, which is known to be a relatively complex disorder. This review analyzes various types of currently existing animal models with different stressors being used. The analysis of this review is based on the criteria of evaluation proposed by Yehuda and Antelman in 1993, as well as the ethical standards of conducting animal studies. While examining the trade-off between the validity of animal models and animal welfare, this review is intended to make a proper evaluation of advantages and limitations of existing animal models and provide innovative insights and possible improvements in the future. As for the animal models mentioned in this review, no single one has attained high validity and ethical standards at the same time, which means there is still progress to be made in future animal studies for PTSD.
\end{abstract}

Keywords: Animal models, PTSD, Electric shock, Underwater trauma, Social defeat, Direct predator exposure, Predator odor exposure

\section{INTRODUCTION}

Post-traumatic stress disorder (PTSD) is a highly distressing mental illness that can be triggered by the past traumatic experiences of an individual. Traumatic experience has become prevalent all over the world. Especially after the outbreak of infectious diseases in the 21st century, the prevalence of PTSD has increased among the general public. A meta-analysis of PTSD rates after Covid-19 outbreak revealed that the estimated PTSD prevalence after the Covid-19 pandemic was $19.3 \%$ among the general public and was $26.9 \%$ among healthcare workers [1]. Although PTSD is affecting more and more people, current treatments are relatively limited. Psychotherapies based on exposure are considered firstline treatments for PTSD, but only $40 \%-60 \%$ of the patients reported that the treatments were helpful [2]. Alongside people who can receive PTSD treatments, there are also people that do not have any access to those treatments. The situation regarding medication for PTSD is even worse. Only 2 types of medications have been approved to treat PTSD by now, while their efficacy is still considered to be limited [2]. Researchers urgently need to find different and more effective treatments. Therefore, more advanced knowledge about the mechanisms of PTSD, both at molecular and neural levels are needed. Animal models have been used in previous researches related to PTSD and other mental health issues. Past examples showed that animal models had contributed a lot to the general understanding of mental health issues, as well as the development of novel treatment. This paper intends to evaluate some representative animal models in order to find out whether there exists an ideal animal model based on previous evaluation criteria and ethical standards for animal research. The animal models were chosen based on types of stressors exerted on the animals, including physical, psychosocial, and predator-related stressors. In terms of both the efficacy of animal models and the welfare of the animals, this paper examines overall advantages and 
limitations of these animal models and provides insights of improvement for animal studies in the field of PTSD in the future.

\section{ANIMAL MODELS AS TRAMSLATIONAL STUDIES OF PTSD}

According to DSM-5, the diagnosis of post-traumatic stress disorder (PTSD) consists of multiple phenotypes, including intrusive thoughts, avoidant behaviors, negative alterations in cognitions and mood, alterations in arousal and reactivity [3]. A few of these symptoms are difficult to be examined in animal studies. For instance, intrusive thoughts are basically studied in terms of selfreport, thus are almost impossible to be measured on animals. Under this circumstance, previous researches focused on other measurable symptoms and have made considerable contributions to the field. Researchers have conducted an analysis of existing animal models findings, which corresponded with various genetic features found in humans with PTSD, involving HPA stress axis, neuropeptides, serotonergic system, and dopaminergic systems [4]. While the analysis demonstrated the significant contributions of established animal models, another study pointed out a need for more vigilant evaluation of the data received in animal studies, because individual variabilities exist and many PTSD symptoms also manifest in other mental health disorders [5]. According to the previous discussions, this paper is designed to provide an overview of the advantages and disadvantages of five animal models that used different types of stressors, based on their validity and ethics.

\subsection{Validity of Animal Models}

To address the validity of animal models used in PTSD researches, Yehuda and Antelman have proposed five criteria of evaluation for useful animal models: the stressors must be able to induce biological and behavioral responses to PTSD after a brief exposure to stressors; induce biological and behavioral responses in an intensity dependent manner; produce biological alterations that can endure for a long time; induce behavioral alterations with bidirectional expression that involve both enhanced and reduced reactivity; be examined with the consideration of interindividual variability [6].
Ethical animal studies should avoid significant harm to the animals. When conducting animal research, researchers must be aware of the welfare of animals and must pursue the best and accurate scientific results, because insufficient pressure may significantly affect the research results [7]. Therefore, whether reaching a balance of these two factors also becomes a criterion of evaluating an animal model.

\section{PHYSICAL STRESSORS}

\subsection{Electrical Shock}

Electrical shock is commonly used in animal studies since it can be administered to an animal in a controllable way. One of the previous studies used 150 male rats as research subjects [8]. The researchers divided these rats into four study groups: those who received shock with situational reminders, shock without situational reminders, no shock with situational reminders, no shock without situational reminders. For the groups receiving shocks, a $2 \mathrm{~mA}$ shock was provided lasting 10 seconds. For the groups receiving situational reminders, the rats were confronted with reminders of that experience of receiving an electrical shock. After the intervention, locomotor activity testing and elevated plus-maze test of anxiety were provided to these rats. The study was designed to investigate behaviors of rats under the influence of electrical shocks and reminders afterward, which was analogous to humans receiving trauma and might provide more insights into understanding PTSD symptoms. When it comes to the first four criteria of Yehuda and Antelman, this study adheres to the standards for high validity. However, it did not demonstrate interindividual variability as Yehuda stated in the fifth criteria relevant to interindividual variability [6]. All the rats studied were male rats, thus not explaining any gender differences among rats while gender may cause a difference in terms of PTSD symptom manifestations. Regarding animal welfare, the electrical shocks received by study subjects were controllable and would not induce huge damages to their health, but were still likely to result in physical injuries. Although there had been risks of endangering the rats already, whether the intensity of electrical shock was enough to have an impact like human trauma remained to be questioned.

\subsection{Ethics of Animal Models}

Table 1. Advantages and Limitations of Various Animal Models of PTSD

\begin{tabular}{|c|c|c|c|c|}
\hline Stressor Types & $\begin{array}{l}\text { Animal } \\
\text { Species }\end{array}$ & Advantages & Limitations & Reference \\
\hline \multicolumn{5}{|c|}{ Physical Stressor } \\
\hline Electrical shock & rat & $\begin{array}{l}\text { 1. reasonable design of stressors } \\
\text { 2. controllable intensity of stressors }\end{array}$ & $\begin{array}{c}\text { 1. may cause physical injures } \\
\text { 2. interindividual variability not well } \\
\text { explained }\end{array}$ & [8] \\
\hline
\end{tabular}




\begin{tabular}{|c|c|c|c|c|}
\hline $\begin{array}{l}\text { Underwater } \\
\text { trauma }\end{array}$ & rat & $\begin{array}{l}\text { 1. explained part of inter-species } \\
\text { variability } \\
\text { 2. no lung damage }\end{array}$ & $\begin{array}{l}\text { 1. interindividual variability not well } \\
\text { explained } \\
\text { 2. uncontrollable intensity of stressors } \\
\text { 3. risk of causing death }\end{array}$ & [9] \\
\hline \multicolumn{5}{|c|}{ Psychosocial Stressor } \\
\hline Social defeat & rat & 1. high construct \& face validity & $\begin{array}{l}\text { 1. interindividual variability not well } \\
\text { explained } \\
\text { 2. uncontrollable intensity of stressors } \\
\text { 3. risk of physical injures }\end{array}$ & [10] \\
\hline \multicolumn{5}{|c|}{ Predator-related Stressor } \\
\hline $\begin{array}{l}\text { Direct predator } \\
\quad \text { exposure }\end{array}$ & rat & $\begin{array}{l}\text { 1. explain interindividual variability to } \\
\text { some extent } \\
\text { 2. relatively natural stressor }\end{array}$ & $\begin{array}{l}\text { 1. high risk of death due to intense } \\
\text { stressor }\end{array}$ & [11] \\
\hline $\begin{array}{l}\text { Predator odor } \\
\text { exposure }\end{array}$ & rat & $\begin{array}{l}\text { 1. in favor of animal welfare } \\
\text { 2. controllable intensity of stressors }\end{array}$ & $\begin{array}{l}\text { 1. interindividual variability not well } \\
\text { explained }\end{array}$ & [12] \\
\hline
\end{tabular}

\subsection{Underwater Trauma}

Underwater trauma is also a commonly used way of simulating human traumatic stress on animals, thus adopted in previous PTSD animal models [9]. Some researchers have been studying the effects of underwater trauma on the response of rats. Normally, rats are placed into an aquarium, firstly swam for 30 seconds, then are submerged into water for another 30 seconds. Researchers then observe and analyze reactions of these rats in terms of follow-up tests. One of the studies particularly used adolescent male rats as study subjects, while most of other studies used adult rats [9]. The interspecies variability can be examined by using samples of different age groups, which according to Yehuda's criteria, is an advantage in terms of validity. The study environment also ensures animal welfare to some extent since the submersion will not cause lung damages to the rats. However, there are still risks of causing death, and in fact, one of the rats being studied died of unknown causes during Moore's study. Furthermore, a lack of interindividual variability appeared in this study with all rats being males. The investigation of gender differences was missing. It was also impossible or difficult for the researchers to control intensity of intervention while adopting the underwater trauma model.

\section{PSYCHOSOCIAL STRESSORS- SOCIAL DEFEAT}

Social stress has been proved to be useful in understanding mechanisms underlying depression, which is one of the major symptoms of PTSD [10]. Rats, as study subjects of this animal model, are placed in the same space with other aggressive rats. Their reactions to the aggressive rats are observed, and social interaction tests are conducted on them afterward. This model possesses high construct validity and face validity, which reaches Yehuda's first, third and fourth criteria of a good animal model, indicating that the stressors produced lasting responses that are both biological and behavioral, and the behavioral responses are bidirectional [6]. In terms of the second criteria, the degree of aggressiveness is hard to control. Interindividual variability is not reflected by this simple design. Additionally, although the model shows social avoidance and the phenotype of depressive symptoms, researchers are still not able to fully capture the underlying molecular mechanisms of PTSD since it also shows symptoms other than depression. Furthermore, there has been a risk of animal wounds in this animal model when the rats are defeated by more aggressive rats, even though the aggressive ones have been screened already before experimental sessions.

\section{PREDATOR-RELATED STRESSORS}

\subsection{Direct Predator Exposure}

To mimic the traumatic experience of humans, predator exposure experiments are also used in animal models and the biobehavioral responses of animals are studied [11]. Previous studies utilizing this type of animal model employed rats and exposed them to predators, which referred to cats in the studies, for 10 minutes. Then researchers tested for behavioral reactions of the studied rats in an elevated plus-maze 7 days after the exposure. Cohen and his colleagues conducted a study using this model in 2003. In addition to previous studies, they randomly assigned exposed rats into two groups representing two extremes, which were exposed well adapted and exposed maladapted, thus enabling them to identify some of the interindividual variability and possibly explain the fact that only a proportion of individuals will develop PTSD after exposure to trauma [11]. This study design increased validity according to Yehuda's criteria, and the stressor was created more naturally compared to physical stressors, such as electrical shocks [6]. However, in terms of ethics, the stressor became relatively intense since being exposed to predators for 10 minutes is not only highly stressful to the rats, but also results in a high risk of death of the study subjects. 


\subsection{Predator Odor Exposure}

Another type of animal model related to predators utilizes predator scent to mimic stressful situations on animals [12]. Instead of being exposed to predators, study subjects are exposed to objects with predator scents on them. For instance, in a study conducted by Cohen and his colleagues in 2006, intervention group rats were placed on litter that soiled with cat odor for 10 minutes, while control group rats were placed on unscented litter for the same amount of time [12]. Afterward, researchers tested several measures of biobehavioral reactions in the elevated plus-maze. The stressor used in this study was less intense than directly exposing rats to the predators, which was more in alignment with animal welfares. Meanwhile, the lack of stressor intensity might have an impact on symptom manifestations. In terms of Yehuda' s validity criteria, this study also did not address interindividual variability at any level [6].

\section{CONCLUSION}

The analysis above demonstrated the advantages and disadvantages of five representative animal models, indicating the lack of ideal animal models in current researches. There exists a trade-off between the validity of studies and animal welfares when people implement animal models by creating various stressors on animals to represent human traumatic events, thus it makes it difficult to design an ideal animal model that reaches high validity and does no harm to animals at the same time. However, the lack of ideal animal models still shows an urgent need for more advanced and refined animal models, which can find a balance between validity and ethics, optimizing the possibility of further understanding of the debilitating mental health issue. For example, since four out of five animal studies mentioned in this review did not address interindividual variability well, researchers need to enhance validity by adding more consideration of factors in the animal models that possibly explain resilience to PTSD of some people, such as gender and age, while minimizing the risks of physical injury of animals. In addition, none of the existing animal models examined all symptoms of PTSD at the same time. Even though some of the symptoms such as intrusive thoughts are hard to manifest on animals, current studies still need more progress with regards to comprehensiveness. Since most current animal studies used rats as study subjects due to their genetic and anatomical similarity to humans, researchers can also conduct more animal studies on other species, such as rabbits. More replications of studies are also needed to increase the reliability of the animal models. Finally, the future direction also includes the utilization of innovative technologies that will assist with the understanding of the pathology and the discovery of biomarkers underlying PTSD, such as the technological advances in neuroimaging including fMRI and MRI.

\section{REFERENCES}

[1] Yuan, K., Gong, Y.-M., Liu, L., Sun, Y.-K., Tian, S.S., Wang, Y.-J., Zhong, Y., Zhang, A.-Y., Su, S.-Z., Liu, X.-X., Zhang, Y.-X., Lin, X., Shi, L., Yan, W., Fazel, S., Vitiello, M. V., Bryant, R. A., Zhou, X.-Y., Ran, M.-S., ... Lu, L. Prevalence of posttraumatic stress disorder after infectious disease pandemics in the twenty-first century, including COVID-19: A meta-analysis and systematic review. Molecular Psychiatry, 2021, pp. 1-17.

[2] Krediet, E., Bostoen, T., Breeksema, J., van Schagen, A., Passie, T., \& Vermetten, E. Reviewing the Potential of Psychedelics for the Treatment of PTSD. International Journal of Neuropsychopharmacology, 23(6) (2020) 385-400.

[3] American Psychiatric Association. Diagnostic and statistical manual of mental disorders (5th ed.), 2013 .

[4] Richter-Levin, G., Stork, O., \& Schmidt, M. V. Animal models of PTSD: A challenge to be met. Molecular Psychiatry, 24(8) (2019) 1135-1156.

[5] Whitaker, A. M., Gilpin, N. W., \& Edwards, S. . Animal Models of Post-Traumatic Stress Disorder and Recent Neurobiological Insights. Behavioural Pharmacology, 25(0) (2014) 398-409.

[6] Yehuda, R., \& Antelman, S. M. Criteria for rationally evaluating animal models of postraumatic stress disorder. Biological Psychiatry, 33(7) (1993) 479486.

[7] Auer, J. A., Goodship, A., Arnoczky, S., Pearce, S., Price, J., Claes, L., von Rechenberg, B., HofmannAmtenbrinck, M., Schneider, E., Müller-Terpitz, R., Thiele, F., Rippe, K.-P., \& Grainger, D. W. Refining animal models in fracture research: Seeking consensus in optimising both animal welfare and scientific validity for appropriate biomedical use. BMC Musculoskeletal Disorders, 8(1) (2007) 72.

[8] Pynoos, R. S., Ritzmann, R. F., Steinberg, A. M., Goenjian, A., \& Prisecaru, I. A behavioral animal model of posttraumatic stress disorder featuring repeated exposure to situational reminders. Biological Psychiatry, 39(2) (1996) 129-134.

[9] Moore, N. L. T., Gauchan, S., \& Genovese, R. F. Differential severity of anxiogenic effects resulting from a brief swim or underwater trauma in adolescent male rats. Pharmacology, Biochemistry, and Behavior, 102(2) (2012) 264-268.

[9] Golden, S. A., Covington, H. E., Berton, O., \& Russo, S. J. A standardized protocol for repeated social defeat stress in mice. Nature Protocols, 6(8) (2011) 1183-1191. 
[10] Cohen, H., Zohar, J., \& Matar, M. The relevance of differential response to trauma in an animal model of posttraumatic stress disorder. Biological Psychiatry, 53(6) (2003) 463-473.

[11] Cohen, H., Zohar, J., Gidron, Y., Matar, M. A., Belkind, D., Loewenthal, U., Kozlovsky, N., \& Kaplan, Z. Blunted HPA Axis Response to Stress Influences Susceptibility to Posttraumatic Stress Response in Rats. Biological Psychiatry, 59(12) (2006) 1208-1218. 